Hans-Jürgen Butt, Ilia V. Roisman, Martin Brinkmann, Periklis Papadopoulos, Doris Vollmer, Ciro Semprebon Current Opinion in Colloid \& Interf. Sci. (2014), 19, pp 343-354

\title{
Characterization of super liquid-repellent surfaces
}

Hans-Jürgen Butt ${ }^{1}$, Ilia Roismann ${ }^{2}$, Martin Brinkmann ${ }^{3}$, Periklis Papadopoulos ${ }^{1}$, Doris Vollmer ${ }^{1}$, Ciro Semprebon ${ }^{3}$

${ }^{1}$ Max Planck Institute for Polymer Research, Ackermannweg 10, D-55128 Mainz, Germany

${ }^{2}$ Center of Smart Interfaces, Technical University Darmstadt, Alarich-Weiss-Straße 10, 64287 Darmstadt, Germany

${ }^{3}$ Max Planck Institute for Dynamics and Self-Organization, Am Faßberg 17, 37077 Göttingen, Germany

Characterization of the wetting properties is a prerequisite for a fundamental understanding and the targeted development of superhydrophobic and superamphiphobic layers. To fabricate super liquid-repellent layers, two requirements need to be met: The surfaces have to be of low energy and their nano- and microstructure needs to be designed in a way that leads to the entrapment of air. The challenge is to design and produce suitable nano- and microstructures to control wetting. Here we describe important methods to quantify wetting properties of super liquid-repellent layers. These properties include the apparent advancing and receding contact angles, the roll-off angle, tensile and lateral adhesion, the impalement pressure, and the observation of drop impact. The most important one is the apparent receding contact angle because it also limits lateral adhesion. The link of these properties to the nano- and microscopic structure of the layer is discussed. Limits, problems, and future challenges are pointed out.

Keywords: Nanostructure, superhydrophobicity, superoleophobicity, superomniphobicity 
Hans-Jürgen Butt, Ilia V. Roisman, Martin Brinkmann, Periklis Papadopoulos, Doris Vollmer, Ciro Semprebon Current Opinion in Colloid \& Interf. Sci. (2014), 19, pp 343-354

\section{Introduction}

Using nano- and microstructured surfaces with low surface energy, scientists have learned to reduce the interaction between liquid and a solid surface. Inspired by the water-repellent properties of some plant leaves and wings of insects we begin to understand the physical principles leading to superhydrophobicity. Superhydrophobicity is based on two principles $[1,2]$ : A low surface energy of the solid surface, often achieved by coating the surface with a fluorinated hydrocarbon, and an increased surface roughness, for example by micropatterning. Low surface energy and high surface roughness lead to the entrapment of air when a drop of water is placed on a superhydrophobic layer. Based on these two characteristic properties many methods to fabricate artificial superhydrophobic layers have been developed (e.g. [3-5]). The challenge is still to invent simple, scalable processes leading to optically transparent, mechanically robust and chemically inert layers. Even greater challenges are to make defect-tolerant or even self-healing superhydrophobic layers.

Shortly after the first superhydrophobic surfaces also oil-repellent layers were made [4]. In the meantime the principle of superamphiphobicity is understood [6-8] and used to produce superamphiphobic layers typically by lithography [7, 9-11] or by self assembly [4, 12-16]. Like for superhydrophobic surfaces one needs to structure the surface in a way that the liquid entraps air. For non-polar liquids, a high roughness is, however, not sufficient. Overhanging structures are required $[6,7,10,17,18]$. The situation, where air is entrapped underneath the drop, is called "Cassie" or fakir state. The Cassie state is a necessary, but not a sufficient requirement for super liquid repellency. In contrast, when the whole solid surface is covered by the liquid the drop is in the "Wenzel" state.

Being able to fabricate liquid-repellent surfaces may open new possibilities both for research and technology. These include self-cleaning, drag reduction [19-22], fog harvesting [23], heat transfer [24], and gas exchange [20, 25]. In microfluidics tiny amounts of liquids can be manipulated with little adhesion and thus little energy dissipation. Therefore, super liquidrepellent surfaces are seen in the broader context which in general aims to control the wetting of surfaces by liquids.

To improve the properties of superhydrophobic and superamphiphobic layers physical methods are required to characterize their wetting properties. The aim of this paper is to 
Hans-Jürgen Butt, Ilia V. Roisman, Martin Brinkmann, Periklis Papadopoulos, Doris Vollmer, Ciro Semprebon Current Opinion in Colloid \& Interf. Sci. (2014), 19, pp 343-354

describe such methods critically, point out limits, open questions, and encourage the discussion on an appropriate standardization. Standardization is important to facilitate the transfer of scientific results into industrial applications, to compare layers produced in different laboratories and to make a comparison to theory easier. Therefore we first need to identify those wetting properties which are relevant and quantifiable. The suggested properties should be linked to a simple and reliable measurement, which can be established in many laboratories and which leads to reproducible results. Furthermore, the outcome of a measurement should be "robust" and not sensitively depend on secondary parameters, which may be difficult to control.

Here, we only discuss wetting properties. One should, however, be aware that for many applications other properties are decisive, such as mechanical stability, chemical inertness and optical transparency. In particular, mechanical properties play an essential role [26-30] because most of the layers with very good wetting properties are not robust enough for e.g. standard scratch test. Here, developing new mechanical test, which take into account the nano- and microscale structure of the layer, would be helpful.

\section{Terminology}

It is useful to first agree on a certain terminology to better organize the vast amount of literature appearing on super liquid-repellency [31]. To our knowledge IUPAC has not defined superhydrophobicity yet. The term comes from the Latin prefix "above", "beyond" (super) and the Greek words for "water" (hydrr) and "fear" (phobos). It is commonly accepted that surfaces, which show a contact angle above $150^{\circ}$ and a roll-off angle of a water drop of less than $10^{\circ}$ are called superhydrophobic. This is not a precise definition. First, one needs to specify, which contact angle is meant, the advancing or receding. Second, the roll-off angle depends on the volume of the drop. Therefore the drop volume needs to be specified. A more accurate definition would be: A layer showing an apparent advancing contact angle of $150^{\circ}$ or higher and a roll-off angle of a drop of distilled water of a specific volume.

If the layers is super oil-repellent, it is called superoleophobic (oleum: Latin for oil) [7]. If the layer repels both, water and oil frequently the term superamphiphobic (amphi: Greek prefix 
Hans-Jürgen Butt, Ilia V. Roisman, Martin Brinkmann, Periklis Papadopoulos, Doris Vollmer, Ciro Semprebon Current Opinion in Colloid \& Interf. Sci. (2014), 19, pp 343-354

for "on both sides", refering to water and oil) is used. In this case a drop of a polar and nonpolar liquid forms a contact angle of $150^{\circ}$ and higher and a roll-off angle lower than $10^{\circ}$ for a drop of specific volume. The liquid needs to be specified. Sometimes the adjective superomniphobic (from Latin omnis "all") is used. As to our knowledge no surface repels perfluorinated alkanes we avoid that term.

With respect to cleaning water, filters have been coated with oil-repellent surfaces. In this case the oil is under water. We talk about under water superoleophobicity. The medium surrounding the oil drop is in this case water rather than air or a gas [32].

In the following we use the term super liquid-repellent layer rather than surface to indicate that it necessarily has a certain extension in the direction normal to the mean surface.

The term "Lotus effect" was to our knowledge first used in 1992 [33] to describe the effect of self-cleaning on plant leaves. The anticontaminant effect of the particular structure of leaves was even pointed out much before. In general, the term Lotus effect is used for the selfcleaning effect on superhydrophobic layers, that are structured on the 0.1 to $100 \mu \mathrm{m}$ length scale, and as a results show a low adhesion to contamination in the form of particles. The contact area between the particles and the superhydrophobic layer is strongly reduced so that contamination is easily rinsed off by water. Self-cleaning and superhydrophobicity sometimes but not always coincide. They are two different phenomena.

\section{Contact angles}

Currently the most widely used parameter to characterize super liquid-repellent surfaces is the contact angle. A high apparent contact angle implies a strong liquid repellency.

Microscopic and macroscopic contact angles. All super-liquid repellent surfaces are structured on the nano- or micrometer length scale. Therefore, it necessary to distinguish between the microscopic contact angles determined by the materials and the macroscopic, apparent contact angles of the layer. We term the contact angle measured on a smooth, homogeneous planar surface of a certain material, the material contact angle $\Theta$. This is the contact angle formed by the liquid when extrapolating the liquid shape on the $10 \mathrm{~nm}-1 \mu \mathrm{m}$ scale to the interface. We neglect the effect of interfacial forces, which may influence the shape of the liquid interface on the $10 \mathrm{~nm}$ scale close to the contact line $[34,35]$. On an 
Hans-Jürgen Butt, Ilia V. Roisman, Martin Brinkmann, Periklis Papadopoulos, Doris Vollmer, Ciro Semprebon Current Opinion in Colloid \& Interf. Sci. (2014), 19, pp 343-354

ideal smooth, inert surface the material contact angle can be described by Young's equation [36]:

$$
\gamma \cos \Theta=\gamma_{S V}-\gamma_{S L}
$$

Here, $\gamma_{1} \gamma_{S V}$, and $\gamma_{S L}$ are the interfacial tensions of the liquid/vapor, solid/vapor, and solid/liquid interfaces, respectively.

It is well known that on real surfaces the contact angle for an advancing liquid is larger than the one for a receding liquid. The advancing contact angle is observed when, for example, the volume of a sessile drop is slowly increased, just before the contact line starts to advance. The receding contact angle is measured when the volume of a sessile drop is decreased just before the contact line recedes. Therefore we distinguish between advancing $\Theta_{a}$ and receding material contact angles $\Theta_{r}$.

The microscopic materials contact angle needs to be distinguished from the macroscopic, apparent contact angle $\Theta^{a p p}$ (Fig. 1). It is the apparent contact angle which is measured by the sessile drop method. The macroscopic scale, that is the length scale observed by eye or with a low-resolution microscope, is much larger than the nano- and microstructures forming the super liquid-repellent layer. Typically it is larger than $10 \mu \mathrm{m}$. In addition to the material properties of the surfaces, $\Theta^{a p p}$ is determined by the nano- and microstructure of the underlying pattern. Again, the apparent advancing contact angle $\Theta_{a}^{a p p}$ is higher than the apparent receding contact angle $\Theta_{r}^{a p p}$. The difference, $\Delta \Theta^{a p p}=\Theta_{a}^{a p p}-\Theta_{r}^{a p p}$, is called apparent contact angle hysteresis. We also distinguish between the three-phase contact line (TPCL), or simply contact line, on the microscopic and the edge of the drop on the macroscopic length scale.

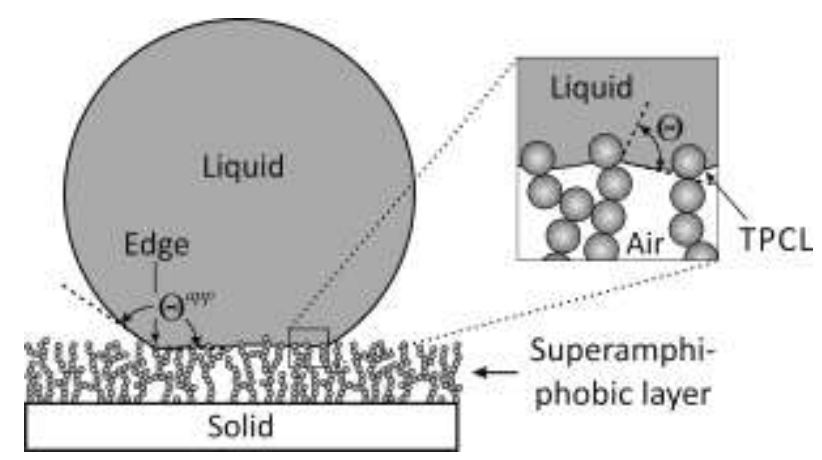


Hans-Jürgen Butt, Ilia V. Roisman, Martin Brinkmann, Periklis Papadopoulos, Doris Vollmer, Ciro Semprebon Current Opinion in Colloid \& Interf. Sci. (2014), 19, pp 343-354

Figure 1. Schematic of a sessile liquid drop on a superamphiphobic surface made of highly porous aggregates of nanospheres. The schematic illustrates the difference between the materials contact angle $\Theta$ and the apparent contact angle $\Theta^{a p p}$. The term "edge" is used to distinguish the apparent contact line and the microscopic three-phase contact line (TPCL).

Measurement of contact angles. Except for few cases [37], in the field of super liquidrepellent layers the contact angle is measured by the sessile drop method. A liquid drop is carefully placed on the super liquid-repellent layer. Its profile is imaged with a camera or a microscope with an illumination behind the drop [38]. Advantages of this technique are its simplicity and the fact that the contact angle is independent on the drop size. In particular for contact angles above $150^{\circ}$, however, the sessile drop method shows systematic errors $[37,39,40]$. Even a tiny deviation of the optical path from the horizontal or tilt to the surface can lead to significant errors in the contact angle. The result strongly depends on details of the experimental setup, such as the used magnification, applied contrast and whether the drop contour is fitted automatically or manually. The results obtained by independent scientists can easily vary up to $5-10^{\circ}$ even if the same setup is used.

The problem is obvious when looking at a laser scanning confocal microscope image of a sessile drops on a super liquid-repellent surface (Fig. 2). The image shows a relatively smooth, gradual increase of the slope of the liquid surface at the contact line. As a result, the apparent contact angle will critically depend on where precisely one places the contact line, the magnification of the video system used to observe the drop, and how the shape is fitted.

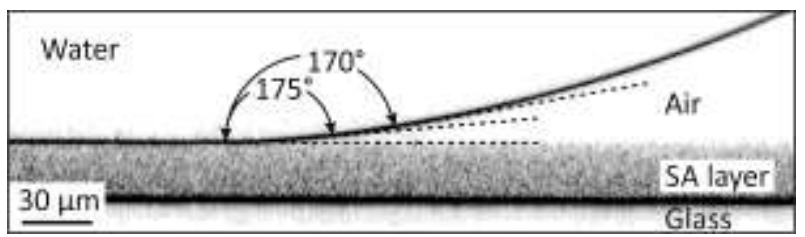

Figure 2. Confocal microscope image of a water drop sitting on a soot-templated superamphiphobic (SA) [15] layer on a glass slide [41]. In this case the reflected rather than fluorescent light was detected. For better visibility the image intensity was inverted. Strong reflection is observed at the top surface of the glass slide and the bottom side of the liquid 
Hans-Jürgen Butt, Ilia V. Roisman, Martin Brinkmann, Periklis Papadopoulos, Doris Vollmer, Ciro Semprebon Current Opinion in Colloid \& Interf. Sci. (2014), 19, pp 343-354

drop. Weak scattering indicates the superamphiphobic layer. The image resolution was about $0.25 \mu \mathrm{m}$ and $1.0 \mu \mathrm{m}$ in the horizontal and vertical directions.

Confocal microscopy $[41,42]$ and interference microscopy $[43,44]$ are alternative ways to measure contact angles of sessile drops more precisely. Both methods are, however, only applicable to transparent samples. Furthermore, confocal and interference microscopes are costly, and so far no commercial data evaluation routines are available.

Often the so called static or as-placed apparent contact angle is reported. The static contact angle is obtained directly after placing a drop on a surface without taking the trouble of expanding and shrinking its volume gradually. The static contact angle is somewhere between the advancing and receding contact angles. It depends on the way the drop is placed onto the surface. Therefore it is only a rough characterization parameter [45]. As argued below, apparent advancing and receding contact angles should be reported. Furthermore, one should be aware that both the advancing and receding contact angle depend on velocity of the edge $v$ [46]. The static advancing and receding angles are those for $v \rightarrow 0$, i.e. the quasistatic limit is taken.

Link between apparent contact angles and the microscopic surface structure. Super liquidrepellency is achieved with two ingredients: appropriate surface chemistry and an appropriate nano- and microstructure. The first requirement is straight forward: The layer needs to have a low surfaces energy, which is typically achieved by coating the layer with fluorinated molecules. To minimize the contact of the liquid with the layer, a high materials contact angle with respective to the liquid is required.

Finding the right nano- and microstructure is the real challenge. Furthermore, there is no such thing as an optimal super liquid-repellent layer, as high apparent contact angles, low adhesion, good mechanical stability, and high slip length cannot be maximized simultaneously. Therefore, the microscopic architecture has to be adapted to the specific function of the layer [47].

One guiding principle to find such an architecture was provided by Cassie and Baxter [48]. For a water drop in thermodynamic equilibrium on a composite (consisting of patches of air 
Hans-Jürgen Butt, Ilia V. Roisman, Martin Brinkmann, Periklis Papadopoulos, Doris Vollmer, Ciro Semprebon Current Opinion in Colloid \& Interf. Sci. (2014), 19, pp 343-354

and solid) surface Cassie and Baxter proposed a simple equation, which relates the apparent contact angle $\Theta^{a p p}$ to the materials contact angle and the surface fraction of solid/liquid interface $\varphi[48-50]$ :

$$
\cos \Theta^{a p p}=\varphi(\cos \Theta+1)-1
$$

Here, 1- $\varphi$ represents the area fraction of trapped air. Although Eq. (2) has been successfully applied to estimate contact angles of some superhydrophobic and even superamphiphobic layers, it is problematic. Liquid drops are often not in thermodynamic equilibrium and their shape is determined by pinning of the contact line [51-56]. They are in a metastable state and not in a global energy minimum. For non-polar liquids on a superamphiphobic layer this metastability is evident; globally the Wenzel state is always favorable. Therefore, Eq. (2) is not applicable to most super liquid-repellent layers.

To illustrate the importance of contact line pinning, we consider a water drop on a square array of rectangular micropillars (Fig. 3a). The material is supposed to be hydrophobic with an advancing contact angle $\Theta_{a}>90^{\circ}$. When focusing on the situation on top of a single pillar and ignoring the precise three-dimensional microscopic shape of the liquid surface (Fig. 3b) the available range for the actual contact angle $\Theta^{a p p}$ is $\Theta_{r} \leq \Theta^{a p p} \leq 90^{\circ}+\Theta_{a}$. For a contact angle below $\Theta_{r}$ the three phase contact line would start to recede and move to the left. For contact angles greater than $90^{\circ}+\Theta_{a}$ the three-phase contact line would start to slide down the side wall of the pillar. In reality the situation is even more complicated and one needs to take into account that the contact line is not straight but distorted [41, 57-59].
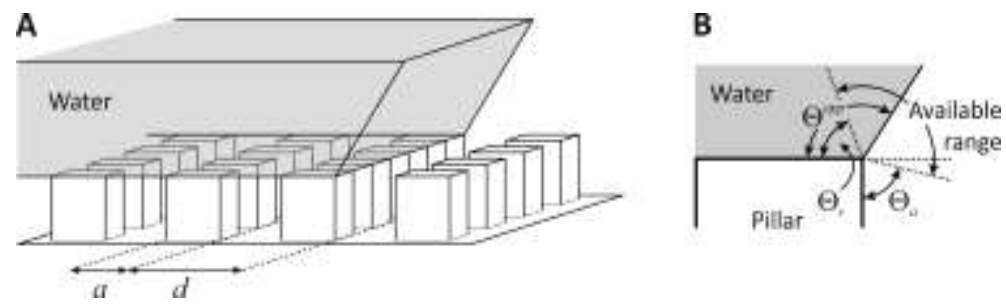

Figure 3. (a) Schematic of a water front on a square array of pillars with a square crosssection. (b) Schematic cross-section of the edge of a drop of water on top of a single hydrophobic pillar. 
Hans-Jürgen Butt, Ilia V. Roisman, Martin Brinkmann, Periklis Papadopoulos, Doris Vollmer, Ciro Semprebon Current Opinion in Colloid \& Interf. Sci. (2014), 19, pp 343-354

Practically the apparent contact angles are, however, limited even to a smaller range. When the volume of the drop is increased the advance of the edge is hindered by the pinning of the contact line on the advancing side of the pillar. For a microarray of square pillars, the average force per unit line of the edge is proportional to $f \propto a \gamma / d$, where $a$ is the width of the pillar and $d$ is the lattice spacing of the array [60]. The apparent contact angle results from the balance of horizontal forces acting on the edge. At an apparent contact angle $\Theta_{a}^{a p p}$ the mean horizontal force component per unit length is $\left|\gamma \cos \Theta_{a}^{a p p}\right|$. This force has to be balanced by the capillary force on the individual pillars. To achieve a high $\Theta_{a}^{a p p}$ this force should be strong. Therefore, the pillars should be as wide as possible and the spacing between pillars should be small so that $a / d \rightarrow 1$. The apparent advancing contact angle cannot exceed $180^{\circ}$ because then the liquid surface would touch the next row of pillars and jump one row. Practically, external vibrations lead to a fluctuation of the liquid surface and a jump to the next row even for contact angles less than $180^{\circ}$. Such vibrations are unavoidable. They happen for example when placing the drop on the surface or increasing the drop volume [61]. When at some place of the edge the liquid jumps to the next row capillary waves are generated, travel along the surface of the liquid and cause fluctuations at the neighboring pillars. Therefore the detachment of the edge at one place can cause detachment/wetting at another [62].

When reducing the volume of a drop the average force per unit line $f$ resisting a receding movement of the edge is also proportional to $a \gamma / d$. To achieve a high apparent receding contact angle this force should be as low as possible. Widely spaced, narrow pillars are favorable. This condition is opposite to the one for a high apparent advancing contact angle. Thus, an optimum has to be found to achieve high advancing and receding contact angles. Apparent advancing and receding contact angles depend on different processes. Therefore, to characterize a super liquid-repellent surface both apparent contact angles are important.

\section{Adhesion}

Tensile adhesion experiments are a complementary way of characterizing liquid-repellency. In an adhesion experiment a small drop of liquid is brought into contact with a super liquidrepellent layer and then gradually withdrawn in normal (vertical) direction. The drop of 
Hans-Jürgen Butt, Ilia V. Roisman, Martin Brinkmann, Periklis Papadopoulos, Doris Vollmer, Ciro Semprebon Current Opinion in Colloid \& Interf. Sci. (2014), 19, pp 343-354

typically $5 \mu \mathrm{L}$ is for example held in a metal ring, a disk, or at the end of a capillary. The force required to pull the drop off the layer, the pull-off or adhesion force, is measured, usually with a microelectromechanical balance [61, 63-65]. Liquid-repellency is correlated with a low adhesion force. Alternatively, the force between a superamphiphobically coated microparticle and a liquid was recently measured using the colloidal probe technique [66].

Fundamentally one can argue via the idealized work of adhesion. "Idealized" in the sense that when separating the liquid from the solid its shape does not change, no surface reconstruction takes place at the solid surface, and the molecular orientation and packing of the liquid molecules does not change (Fig. 4). When separating a liquid from a solid surface net free liquid and free solid surface is created, while solid/liquid interfaces is destroyed. The net change in interfacial energy per unit area is $\gamma+\gamma_{S V}-\gamma_{S L}$. Replacing $\gamma_{S V}-\gamma_{S L}$ by $\gamma \cos \Theta$ one obtains the work of adhesion per unit area: $w=\gamma(1+\cos \Theta)[67,68]$. The actual work of adhesion is certainly different and so far it is unclear how to relate the adhesion force and the work of adhesion. E.g. one assumption in the simple energy balance is that all interfaces are planar. For a liquid this is not fulfilled. Still, it is worthwhile relating the tensile adhesive force to $\gamma(1+\cos \Theta)$. When a drop is withdrawn form a super liquid-repellent layer its edge recedes. Therefore, the apparent receding contact angle $\Theta_{r}{ }^{a p p}$ should be used [31]. Indeed, Samuel found experimentally a linear increase of the pull-off force for aqueous drops versus $\gamma\left(1+\cos \Theta_{r}^{a p p}\right)[61]$.

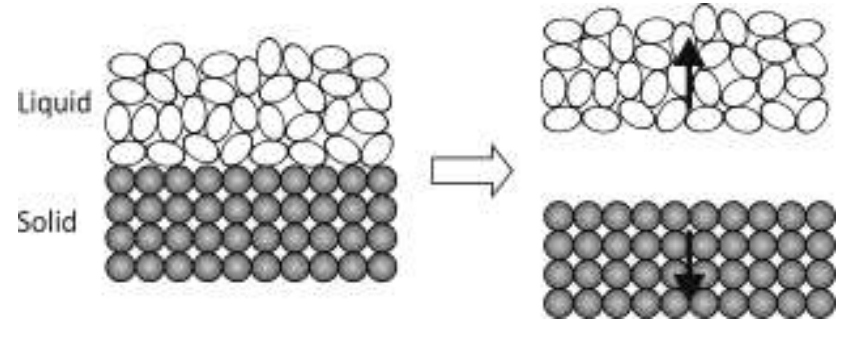

Figure 4. Idealized thought experiment of separating a liquid from a solid and creating fresh solid and liquid surface at the expense of solid/liquid interface. 
Hans-Jürgen Butt, Ilia V. Roisman, Martin Brinkmann, Periklis Papadopoulos, Doris Vollmer, Ciro Semprebon Current Opinion in Colloid \& Interf. Sci. (2014), 19, pp 343-354

Adhesion measurements have the advantage of being very sensitive. Keeping the drop size constant, different samples can be compared within one device. The drawback of this method is that in general the work of adhesion is affected by the actual shape of the liquid drop, which is related to the size of the ring and to the liquid volume. As a consequence a quantitative analysis linking the adhesion force to material properties, like e.g. the receding contact angle, is more involved and systematic calculations are still missing. Axisymmetric drop profiles, which are solutions of the Laplace equation can be described by a class of axisymmetric constant mean curvature surfaces termed unduloids or nodoids (in the absence of gravity). By matching the boundary conditions it is possible to analytically determine the force curves. Baret and Brinkmann [69] investigated the mechanisms for the instability of such shapes, and determined the condition under which, when depositing a sessile drop with a syringe, it can stick to the substrate.

Knowledge of the drop shape allows a direct calculation of the attractive force. As an example we calculated the capillary force during one cycle of a typical adhesion experiment (Fig. 5a). A drop is hanging from a circular disk of radius $a$. We neglect gravitation. The top contact line of the drop is pinned at the edge during the whole experiment and initially is assumed to form an angle of $120^{\circ}$ ( 1 in Fig. 5a). From the spherical cap geometry the volume of the drop is given as $V=5.44 a^{3}$. When lowering the disk at some point the drop will get into contact with the super liquid-repellent layer. With the initial top angle of $120^{\circ}$, contact will be established at $h=1.73 a$. We assumed that directly after contact the bottom edge of the drop jumps to a contact angle similar to $\Theta_{a}^{a p p}(2)$. As a result the angle of initially $120^{\circ}$ will slightly change.

Once contact has been established a capillary force is acting between the top disk and the substrate [70]. It consist of two parts: The direct action of the surface tension along the lower edge, $2 \pi r \gamma \sin \Theta_{a}^{a p p}$ plus the Laplace pressure $\Delta P$ acting over the contact area $\pi r^{2} \Delta P$. In our example we assume that the lowering of the disk is stopped directly after contact has been established. Then the disk is gradually moved upward again, implying that the $\Theta^{a p p}$ decreases. The disk is moved so slowly that equilibrium is reached at all times. In Fig. 5B we plot the capillary force for constant contact radius $r$ (dashed lines). Each radius is determined by setting the advancing contact angles $\Theta_{a}^{a p p}$ of $130^{\circ}, 140^{\circ}, 150^{\circ}$, or $150^{\circ}$ when the drop 
Hans-Jürgen Butt, Ilia V. Roisman, Martin Brinkmann, Periklis Papadopoulos, Doris Vollmer, Ciro Semprebon Current Opinion in Colloid \& Interf. Sci. (2014), 19, pp 343-354

touches. Increasing the separation $h$ the apparent contact angle on the super liquidrepellent layer decreases; please note that the contact angle indicated on the dashed lines are only the initial contact angles directly after contact.

Once the actual apparent contact angle has reached the apparent receding angle, $\Theta^{a p p} \rightarrow \Theta_{r}^{a p p}$, the edge starts to recede at constant contact angle, namely at $\Theta^{a p p}=\Theta_{r}^{a p p}$ (Fig. $5 A, 3)$. Capillary forces calculated for different constant contact angles are plotted as continuous lines in Figure 5B. The slope of plot of capillary force-versus-distance is lower for constant contact angle than constant contact radius. The main reason is that for constant contact radius the curvature of the liquid surface and $\Delta P$ changes more than for a sliding edge.

Finally the liquid bridge becomes unstable and, for the range of material angles considered here, it detaches from the layer ( 4 in Fig. 5a). The condition for the detachment changes. For detachment it is relevant whether the experiment is carried out at fixed pulling force or at fixed distance $h$. The reason is that the adhesion force-versus-distance plot may show a maximum. When pulling with regulated, gradually increasing force, the drop detaches at the maximum of this force-versus-distance graph. When pulling with regulated, gradually increasing distance, detachment occurs at a lower force but larger distance.

A typical example for an adhesion force measurement is indicated in dark grey in Figure 5B. This specific example is for $\Theta_{a}^{a p p}=150^{\circ}, \Theta_{r}^{a p p}=140^{\circ}$, and $h=1.73 a$. We supposed that the drop initially attains a contact radius $r$ determined by $\Theta_{a}{ }^{a p p}$ when getting into contact at $h=1.73 a$ with the super liquid-repellent surface (bottom left of the dark grey curve in Fig. 5B). When increasing $h$ the edge remains pinned and the force increases roughly linearly with $h$. At $h=1.84 a$ the apparent contact angle reaches $\Theta_{r}{ }^{a p p}$ and the curve starts to follow the branch of constant (receding) contact angle. The edge recedes at constant contact angle of $140^{\circ}$. The attractive force reaches a maximum at $h=1.86 a$ and decreases afterwards. At $h=1.94 a$ the liquid bridge becomes unstable and the drop is released from the super liquidrepellent layer.

Figure $5 \mathrm{C}$ shows capillary forces for three different scaled volumes of the drop. We see three effects: with increasing volume the scaled distance, where the drop touches the surface, 
Hans-Jürgen Butt, Ilia V. Roisman, Martin Brinkmann, Periklis Papadopoulos, Doris Vollmer, Ciro Semprebon Current Opinion in Colloid \& Interf. Sci. (2014), 19, pp 343-354

increases; the adhesion force increases; the maximum of the force curve shifts from the constant contact radius to constant contact angle branch. Therefore, the volume changes the force qualitatively.

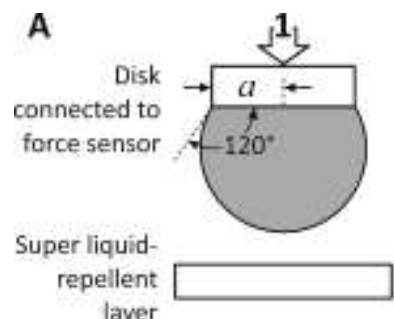

2

layer
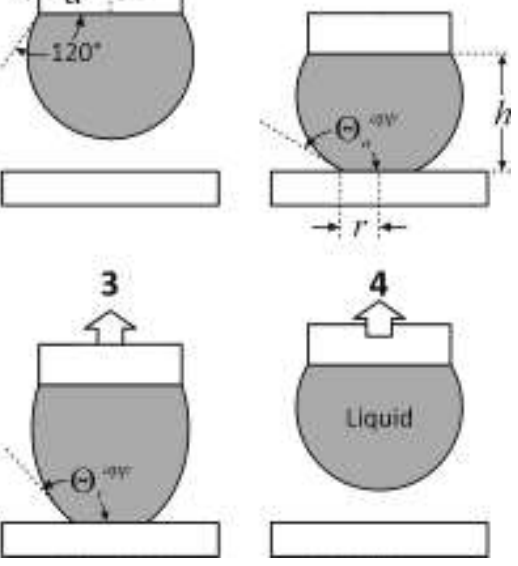

4
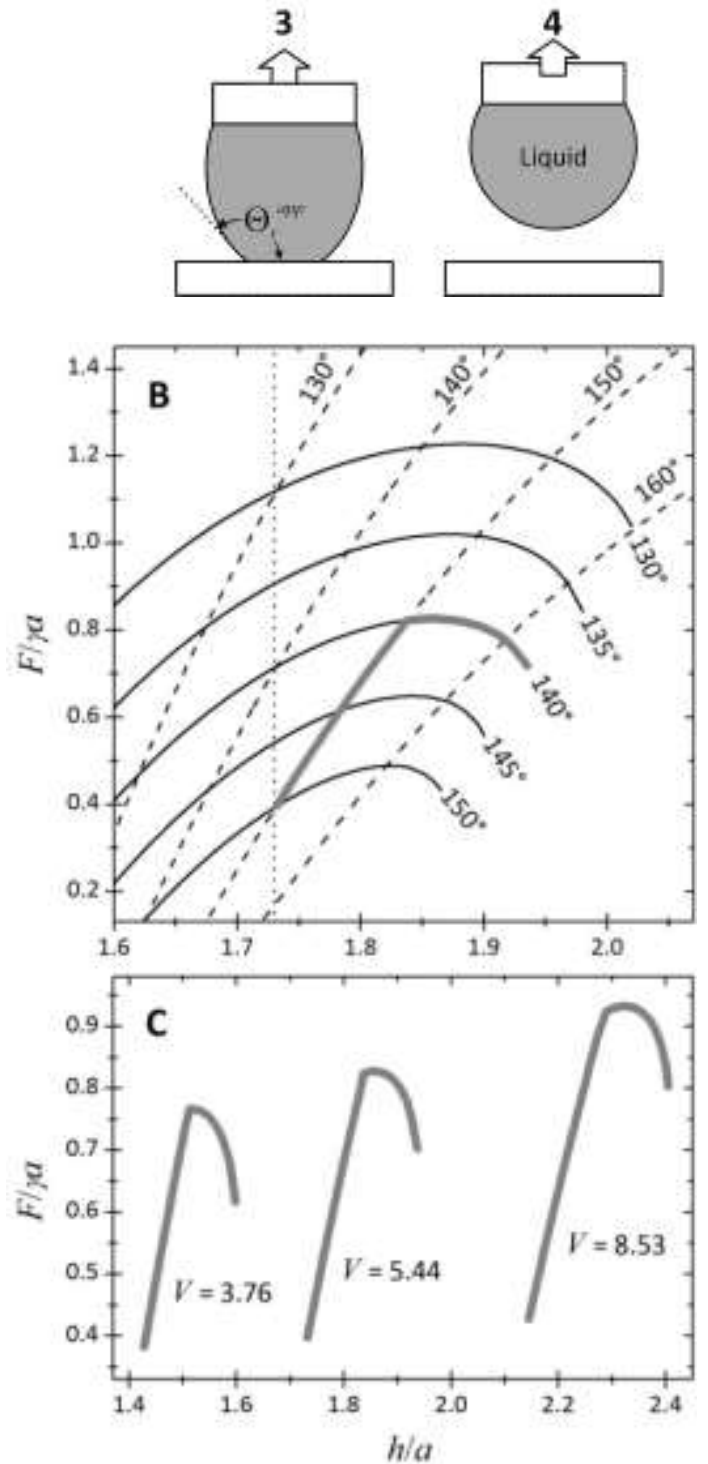

Figure 5. A: Schematic of an adhesion force measurement. B, C: Calculated attractive capillary force $F$, scaled by the surface tension $\gamma$ and the contact radius at the top disk $a$ versus the distance $h$ scaled by the contact radius $a$. The system is rotational symmetric. B: 
Hans-Jürgen Butt, Ilia V. Roisman, Martin Brinkmann, Periklis Papadopoulos, Doris Vollmer, Ciro Semprebon Current Opinion in Colloid \& Interf. Sci. (2014), 19, pp 343-354

Fixed drop volume $V=5.44 a^{3}$ for constant contact radii (dashed) and constant contact angle (continuous, apparent advancing contact angle $130,135,140,145^{\circ}$, and $150^{\circ}$ ). Dashed lines describe forces where the contact line is pinned to a fixed radius. For pinned contact line the contact radius $r$ was calculated from $\Theta_{a}{ }^{a p p .}=130^{\circ}, 140^{\circ}, 150^{\circ}$, and $160^{\circ}$. The vertical dotted line indicates the distance at which the drop contacts the layer. C: Scaled force-versus-scaled distance for drop volumes of $V=3.76,5.44$ and $8.53 a^{3}$. The curves were calculated for a contact radius given by an initial contact angle of $150^{\circ}$ and constant contact angle once the apparent contact angle has reached $\Theta_{r}^{a p p}=140^{\circ}$.

\section{Roll-off angle}

Measuring roll-off angles. The second parameter often used to characterize super liquidrepellent surfaces is the roll-off also called sliding or slip angle $\alpha[4,71]$. Measuring roll-off angles is a well-known method to measure lateral adhesion. This technique is referred to as the tilted plane method [67, 72-79]. A drop of the liquid is placed on a horizontal surface. Then the surface is gradually tilted. At the roll-off angle $\alpha$ the drop rolls off the surface. The lower the roll-off angle, the lower the lateral adhesion between liquid and solid and the stronger the liquid repellency.

For a quantitative analysis the lateral adhesion force caused by pinning of the three-phase contact line, $F_{L A}$, is set equal to the lateral component of the gravitational force, $V \rho g \sin \alpha$. Here, $V$ is the volume of the drop, $\rho$ is the density of the liquid, $g=9.81 \mathrm{~m} / \mathrm{s}^{2}$ is the acceleration of gravity. $F_{L A}$ is proportional to the surface tension of the liquid, the length of the edge and the difference of the cosines of the apparent contact angles at the leading and trailing edges $\cos \Theta_{r}^{a p p}-\cos \Theta_{a}^{a p p}[67,74,78,80]$. As a result we get

$$
\sin \alpha=k \frac{\gamma r}{V \rho g}\left(\cos \Theta_{r}^{a p p}-\cos \Theta_{a}^{a p p}\right)
$$

Here, $r$ is the equivalent radius of a circle with the same area as the apparent contact area and $k$ is a constant, which depends on the precise conditions; it has been calculated to be between $k=4 / \pi$ and $2.0[73,75,76,78,81]$. Theoretically, for low hysteresis, which is the 
Hans-Jürgen Butt, Ilia V. Roisman, Martin Brinkmann, Periklis Papadopoulos, Doris Vollmer, Ciro Semprebon Current Opinion in Colloid \& Interf. Sci. (2014), 19, pp 343-354

case on super liquid-repellent surfaces, $k=4 / \pi=1.27$ is the best value while $k=2$ is for strong hysteresis [82]. Experimentally a value of $k=1.9$ seems to be a good choice for water on different polymer surfaces, even for low hysteresis $[62,73,82,83]$. Since the apparent contact radius is proportional to $V^{1 / 3}$ the sine of the roll-off angle is proportional to $\sin \alpha \propto V^{-2 / 3}$. The roll-off angle decreases with increasing volume of the drop. Therefore, when roll-off angles are reported also the volume of the drop needs to be given. A suitable volume is that of a drop of the liquid with a diameter corresponding to the capillary length $\kappa=\sqrt{\gamma / g \rho}$ or $V=\pi \kappa^{3} / 6$. For water this volume would by $10.3 \mu \mathrm{L}$.

Practically $\Theta_{r}{ }^{a p p}$ is more relevant than $\Theta_{a}^{a p p}$ simply because the cosine of the angle changes less and less when approaching $180^{\circ}$. Thus going from $160^{\circ}$ to $170^{\circ}$ only leads to a change in cosine of 0.045 while going from $140^{\circ}$ to $150^{\circ}$ leads to a change of 0.10 .

Relation between roll-off angle, $\Theta_{a}^{a p p}$, and $\Theta_{r}^{a p p}$. Reporting the roll-off angle together with the volume of a drop is a good way of characterizing a super liquid-repellent layer. When however, trying to relate contact angle hysteresis quantitatively to the roll-off angle one needs to consider several possible effects and problems:

- For very high contact angles the apparent contact radius, $r$, is difficult to measure precisely by the sessile drop method. For drops which are much smaller than the capillary length $\sqrt{\gamma / g \rho}$, so that they are spherically shaped, $r$ can be calculated from the volume by [76]

$$
r=\left[\frac{V}{\pi} \frac{3 \sin ^{3} \Theta^{a p p}}{\left(1-\cos \Theta^{a p p}\right)^{2}\left(2+\cos \Theta^{a p p}\right)}\right]^{1 / 3}=\left[\frac{6 V}{\pi} \frac{1}{\tan \left(\Theta^{a p p} / 2\right)\left[3+\tan ^{2}\left(\Theta^{a p p} / 2\right)\right]}\right]^{1 / 3}
$$

Here, we assumed that the contact angle hysteresis is small so that in Eq. (4) we can set $\Theta_{r}^{a p p} \approx \Theta_{a}^{a p p}=\Theta^{a p p}$. In reality the apparent contact area is not perfectly circular but is elongated along the sliding direction. As a first approximation it can be taken as elliptical [78]. To obtain both axes two cameras are needed.

- Krasovitski and Marmur point out that on patterned substrates the contact angle at the upper, trailing edge of the drop at the stability limit $\Theta_{\min }$ and the contact angle at the lower, leading edge of the drop just before roll-off $\Theta_{\max }$ are not necessarily equal to the 
Hans-Jürgen Butt, Ilia V. Roisman, Martin Brinkmann, Periklis Papadopoulos, Doris Vollmer, Ciro Semprebon Current Opinion in Colloid \& Interf. Sci. (2014), 19, pp 343-354

apparent receding and advancing contact angles, respectively [84]. In most cases, however, within experimental error margin $\Theta_{\min }=\Theta_{r}{ }^{a p p}$ and $\Theta_{\max }=\Theta_{a}{ }^{a p p}$ for super-liquid repellent surfaces.

- It has been observed by Pierce et al [85] that different protocols can lead remarkably different measures of roll-off angles. Recently Semprebon and Brinkmann [86] pointed out that these differences can be related to the quasi static deformation of the drop before the onset of motion. Therefore for a given substrate there is a certain range of possible roll-off angles, whose width scales with the range of apparent contact angle hysteresis.

To estimate the roll-off angle it is sufficient to report the apparent receding contact angle of a super liquid-repellent layer. The reason is that for a given receding contact angle, the rolloff angle cannot exceed a certain threshold. To calculate this threshold one may insert $\Theta_{a}^{a p p}$ $=180^{\circ}$ in Eq. (3), because $\Theta_{a}^{a p p}=180^{\circ}$ leads to the largest possible difference in $\cos \Theta_{r}^{a p p}-\cos \Theta_{a}^{a p p}$ and thus to the largest possible roll-off angle.

This, however, only leads to a rough estimate. A more precise upper limit for $\alpha$ is obtained when considering the influence of the contact angle on the contact radius. For super-liquid repellent surfaces the contact angle hysteresis is low $\left(\Theta_{a}^{a p p}-\Theta_{r}^{a p p} \leq 30^{\circ}\right.$ [76]). To calculate this maximal roll-off angle from Eq. (3) we use the mean contact angle $\left(\Theta_{r}^{a p p}+\Theta_{a}^{a p p}\right) / 2=\Theta^{a p p}$ in Eq. (4) to calculate the contact radius. Then we need to find the maximum of

$$
\sin \alpha=k\left(\frac{6}{\pi}\right)^{1 / 3} \frac{\gamma}{V^{2 / 3} \rho g} \frac{\cos \Theta_{r}^{a p p}-\cos \left(\Theta_{r}^{a p p}+\Delta \Theta\right)}{\tan ^{1 / 3}\left(\frac{\Theta_{r}^{a p p}+\Delta \Theta / 2}{2}\right)\left[3+\tan ^{2}\left(\frac{\Theta_{r}^{a p p}+\Delta \Theta / 2}{2}\right)\right]^{1 / 3}}
$$

This function is maximal for $\Delta \Theta \approx 0.55\left(180^{\circ}-\Theta_{r}^{a p p}\right)$. Maximal roll-off angles for water drops on surfaces with receding contact angles of $140^{\circ}, 150^{\circ}$, and $160^{\circ}$ calculated with Eq. (5) and $\Delta \Theta \approx 0.55\left(180^{\circ}-\Theta_{r}^{a p p}\right)$ are plotted in Figure 6.

Note, that for fixed apparent receding contact angle the roll-off angle decreases with decreasing interfacial tension. This implies that a drop of hexadecane rolls off more easily 
Hans-Jürgen Butt, Ilia V. Roisman, Martin Brinkmann, Periklis Papadopoulos, Doris Vollmer, Ciro Semprebon Current Opinion in Colloid \& Interf. Sci. (2014), 19, pp 343-354

than a drop of water. However for an identical layer, hexadecane shows a lower apparent receding contact angle than water, in line with the observation that the apparent receding contact angle decreases with decreasing interfacial tension [7].

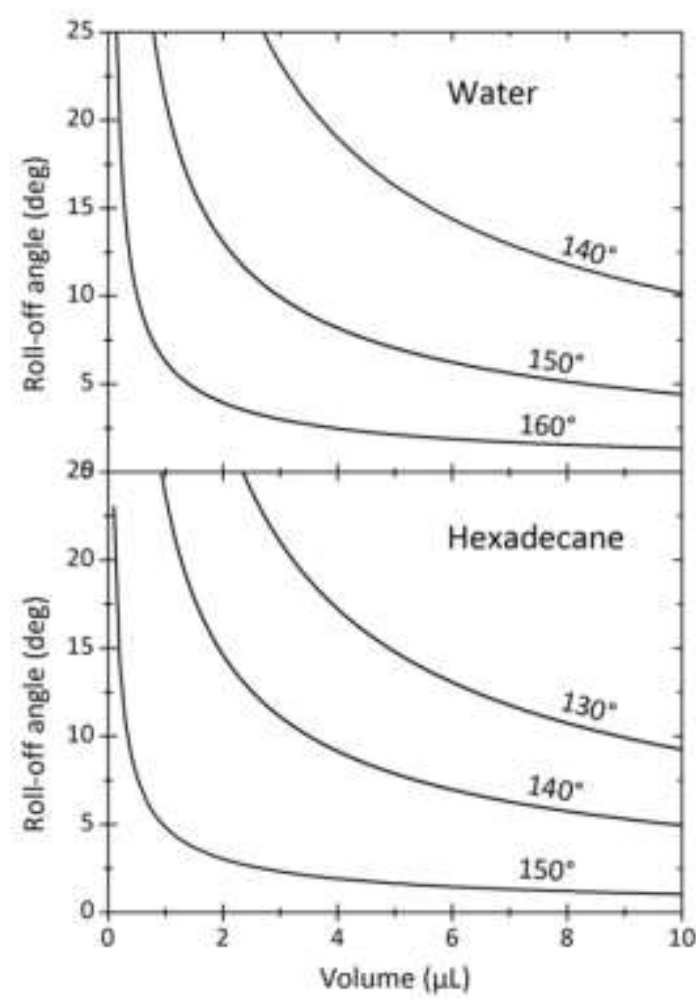

Figure 6. Maximal roll-off angles possible for a drop of water $\left(\rho=997 \mathrm{~kg} / \mathrm{m}^{3}, \gamma=0.0720 \mathrm{~N} / \mathrm{m}\right)$ and hexadecane $\left(\rho=770 \mathrm{~kg} / \mathrm{m}^{3}, \gamma=0.0271 \mathrm{~N} / \mathrm{m}\right)$ at $25^{\circ} \mathrm{C}$ at a given receding apparent contact angle of $130^{\circ}, 140^{\circ}, 150^{\circ}$, and $160^{\circ}$ versus the drop volume. A spherical drop shape was assumed and Eqs. (3-4) with $\Delta \Theta \approx 0.55\left(180^{\circ}-\Theta_{r}^{a p p}\right)$ were applied. Above the plotted lines the drop certainly rolls off the layer.

\section{Impalement}

A necessary requirement for super liquid-repellency is that the liquid is in the Cassie state with air trapped underneath. When the liquid impales the layer and goes to the Wenzel state the contact angle hysteresis increases and super liquid-repellency is lost. The critical pressure at which the liquid changes from the Cassie to the Wenzel state is the impalement 
Hans-Jürgen Butt, Ilia V. Roisman, Martin Brinkmann, Periklis Papadopoulos, Doris Vollmer, Ciro Semprebon Current Opinion in Colloid \& Interf. Sci. (2014), 19, pp 343-354

pressure. A penetration of the layers needs to be prevented because usually the transition is irreversible. Once a non-polar liquid has impaled a superamphiphobic layer, the whole liquid has to be removed, e.g. by evaporation, before the super-liquid repellent state can be reached again.

Impalement mechanisms. Two main pathways for impalement have been discussed: depinning and sagging $[11,17,42,47,50,51,87-92]$. To illustrate them let us consider water on a square array of cylindrical, hydrophobic pillars of diameter $a$ and a pillar-to-pillar distance $d$. For negligible gravity the water surface would be perfectly planar and the crosssection would be a straight line (Fig. 7a). When a static pressure is applied, for example the hydrostatic pressure, the liquid will start to sag in between the pillars. Due to the resulting curvature a capillary counter pressure stabilizes the liquid. The pressure is directly linked to the curvature by the Laplace equation.

When increasing the pressure and depending on $a, d$, and the pillar height $h$ the water will impale the layer in different ways. For low pillars the bottom surface of the liquid will sag in the layer until it touches the substrate (Fig. 7b) $[87,88]$. Then it will spread sideways and flood the whole superhydrophobic structure. If we, for example consider a square array of micropillars, the deepest point on the liquid surface $\left(h-h_{\text {sag }}\right)$ is on the diagonal between two pillars [42]. The precise shape of the liquid surface has to be calculated numerically [93]. As a rough approximation, we describe the cross-section of the liquid by a segment of a circle. Then the critical height where the drop touches the substrate $h_{\text {sag }}=h$ is given by

$$
h=\frac{d}{\sqrt{2}} \tan \left(\frac{\Theta-90^{\circ}}{2}\right) h=\frac{d}{\sqrt{2}} \frac{1-\cos \left(\Theta-90^{\circ}\right)}{\sin \left(\Theta-90^{\circ}\right)}=\frac{d}{\sqrt{2}} \tan \left(\frac{\Theta-90^{\circ}}{2}\right)
$$

Here, $\Theta \leq \Theta_{a}$ is the actual microscopic contact angle. The radius of curvature of the liquid surface along the diagonal is roughly $\left[h^{2}+d^{2} / 2\right] / 2 h$, leading to a Laplace pressure of

$$
P_{\text {sag }}=\frac{4 \gamma h}{h^{2}+d^{2} / 2} \quad \text { for } \quad h \leq \frac{d}{\sqrt{2}} \tan \left(\frac{\Theta_{a}-90^{\circ}}{2}\right)
$$

For sufficiently high pillars the liquid will sag until it reaches the microscopic advancing angle. The situation becomes unstable and the liquid will impale the layer (Fig. 7c). The 
Hans-Jürgen Butt, Ilia V. Roisman, Martin Brinkmann, Periklis Papadopoulos, Doris Vollmer, Ciro Semprebon Current Opinion in Colloid \& Interf. Sci. (2014), 19, pp 343-354

radius of curvature along the diagonal is given by. $d /\left[\sqrt{2} \sin \left(\Theta_{a}-90^{\circ}\right)\right]$. With $\sin \left(\Theta_{a}-90^{\circ}\right)=-\cos \Theta_{a}$ we get a maximal supported pressure of

$$
P_{\text {sag }}=-2 \sqrt{2} \frac{\gamma}{d} \cos \Theta_{a} \text { for } \quad h>\frac{d}{\sqrt{2}} \tan \left(\frac{\Theta_{a}-90^{\circ}}{2}\right)
$$

Once the applied pressure is higher than $P_{s a g}$, the three-phase contact line depins from the edge of the pillars, the liquid surface starts to slide down the walls of the pillars and the layer is impaled. During this process the actual microscopic contact angle with the walls of the pillars is equal to $\Theta_{a}$ (Fig. 7d) [94]. The capillary force per pillar is equal to the circumference $\pi a$ times the vertical component of the surface tension $\gamma\left|\cos \Theta_{a}\right|[1,95]$. Dividing this force by the area per pillar leads to an impalement pressure of

$$
P_{d e p}(1-\varphi)=-\frac{\pi a \gamma}{(d+a)^{2}} \cos \Theta_{a}
$$

For $P_{s a g}<P_{d e p}$ sagging is more likely while for $P_{s a g}>P_{d e p}$ the Cassie-to-Wenzel transition occurs via depinning.

We would like to add another way of impalement which is important for long-term stability of volatile liquids: Evaporation and condensation (Fig. 7e). Due to the curvature underneath a liquid on top of a super liquid-repellent surface the vapor pressure is increased according to the Kelvin equation. If the vapor cannot equilibrate with the surrounding it may start condensing in corners and on the substrate. The amount of condensed liquid increases due to transfer of molecules through the vapor phase. 
Hans-Jürgen Butt, Ilia V. Roisman, Martin Brinkmann, Periklis Papadopoulos, Doris Vollmer, Ciro Semprebon Current Opinion in Colloid \& Interf. Sci. (2014), 19, pp 343-354

(a)

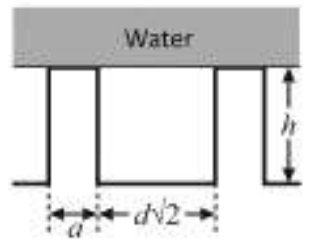

(c)

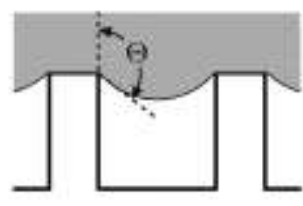

(e)

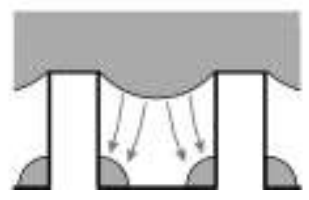

(b)

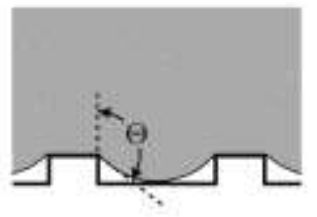

(d)

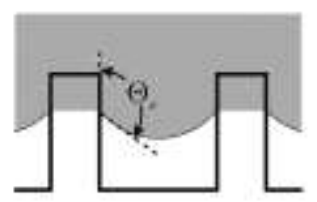

Figure 7. Schematic cross-section through the diagonal of an array of cylindrical, hydrophobic pillars of height $h$, diameter $a$, and lattice constant $d+a$ with water on top. (a) At zero pressure; (b) Sag impalement for low pillar height; (c) Sag impalement for high pillars; (d) Depinning impalement; (e) Impalement due to evaporation and condensation.

Measuring impalement. Measuring the impalement pressure is far from being a standard method. One way is to watch an evaporating water drop. While the drop evaporates its radius decreases and its capillary pressure $P=2 \gamma / R$ increases; here, $R$ is the radius of the drop. When the capillary pressure is so high, that is exceeds the impalement pressure the drop changes from the Cassie to the Wenzel state $[11,88,89,94]$. This transition can usually be observed by a jump in the contact area. If the edge is fixed and does not jump one can still observe a transition from constant contact angle to constant contact radius evaporation [42]. The method is, however, limited to relatively low impalement pressures because the drop needs to have a certain size; otherwise the edge is difficult to observe by optical microscopy and the drop evaporates within seconds. For a minimal drop size of say $R=50$ $\mu \mathrm{m}$ the corresponding maximal detectable impalement pressure is $\approx 3 \mathrm{kPa}$. To reach higher 
Hans-Jürgen Butt, Ilia V. Roisman, Martin Brinkmann, Periklis Papadopoulos, Doris Vollmer, Ciro Semprebon Current Opinion in Colloid \& Interf. Sci. (2014), 19, pp 343-354

pressures and study long-term stability of entrapped air, Poetes et al. [96] immersed superhydrophobic plates in a water tank of up to $1.5 \mathrm{~m}$ depth, reaching a pressure of $1.5 \times 10^{4} \mathrm{~Pa}$.

For transparent super-liquid-repellent surfaces one can use an interference [97] or confocal microscope [42] and record the pressure where the entrapped air disappears. Entrapped air can be detected with high sensitivity due to the large difference in refractive index between condensed matter and gas. Therefore, plastrons scatter light effectively.

Pressing on a drop is another way of inducing impalement [45, 87, 98, 99]. Therefore, a liquid drop is deposited onto the horizontal super liquid-repellent layer. Another horizontal plate is moved downwards until the drop is compressed. This plate may be coated with the same layer as the bottom substrate (Fig. 8) [87]. Care must be taken to prevent the drop from sliding out off the gap. The liquid is squeezed sideways and the Laplace pressure in the liquid increases. The Laplace pressure is given by the curvature of the liquid by $P=\gamma(1 / R+1 / l) \approx \gamma / R$. Here, $R$ is the radius in vertical direction and $l$ the radius in horizontal direction. We can relate the thickness of the liquid layer $h$ to the apparent advancing contact angle of the super liquid-repellent layer: $h=-2 R \cos \Theta_{a}^{a p p}$. Here, we assumed that the horizontal extension of the liquid is much larger than the layer thickness $(l$ > h) and we assumed a circular shape of the liquid surface (circular approximation). In the experiment the drop is compressed until it impales the super liquid-repellent layer. With the Laplace pressure $P=\gamma / R$ we can calculate the impalement pressure from

$$
P_{i m p}=-\frac{2 \gamma}{h} \cos \Theta_{a}^{a p p}
$$

The challenge is to measure precisely the contact angles and the gap thickness. This may prove difficult because again the optical path would need to be perfectly horizontal.

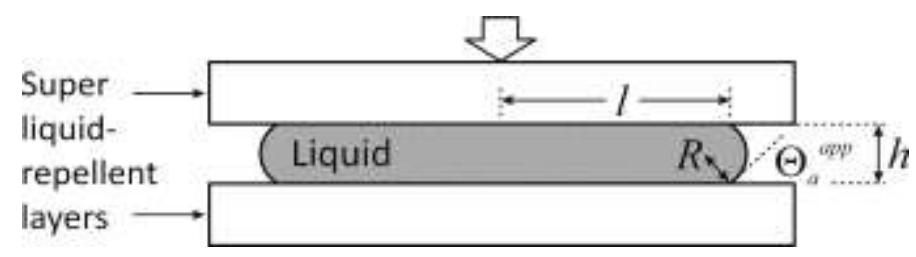

Figure 8. Schematic of a drop pressed between two similar super liquid-repellent plates. 
Hans-Jürgen Butt, Ilia V. Roisman, Martin Brinkmann, Periklis Papadopoulos, Doris Vollmer, Ciro Semprebon Current Opinion in Colloid \& Interf. Sci. (2014), 19, pp 343-354

An alternative to the optical method would be to measure the force exerted onto the plate. This force is given by the direct action of the surface tension of the liquid plus the capillary pressure acting over an area $\pi l^{2}$ :

$$
F=2 \pi \gamma l \sin \Theta_{a}^{a p p}+\pi l^{2} P
$$

Usually, the second term is much larger than the first one and

$$
F=-\frac{2 \pi l^{2} \gamma}{h} \cos \Theta_{a}^{a p p}
$$

From a measurement of $F, l$ and $h$ one can calculate the impalement pressure. Impalement should be visible from a full $F$-vs- $h$ curve.

\section{Dynamic characteristics of superhydrophobic surfaces: drop impact}

One possibility to obtain information on the properties of super liquid-repellent layers is to study the outcome of drop impact. Impact dynamics of liquid drops is directly relevant for self-cleaning and a number of industrial processes such as rapid spray cooling, spray painting and coating $[100,101]$. When coating windows or solar cells with a super liquid-repellent layer for self-cleaning the impacting rain drops should not impale the layer.

In a drop impact experiment a drop of defined volume and initial velocity collides with a super liquid-repellent layer. Drop impact is observed using a high-speed video camera. The interfacial tension, the viscosity of the liquid, and the chemical and physical properties of the surface and its layer structure determine the impact dynamics. The dynamics of drop impact was investigated for different impact velocity and drop sizes on regular and random superhydrophobic surfaces [102-109]. For superamphiphobic layers drop impact experiments are even more important since impalement of non-polar liquids leads to a permanent breakdown of the Cassie state $[106,110,111]$.

Drop impact is usually described as a function of the dimensionless Weber and Reynolds number. The Weber number represents the ratio of the kinetic to the surface energy: $W e=\rho D_{0} U_{0}^{2} / \gamma$. The Reynolds number, $R e=\rho D_{0} U_{0} / \eta$, is the ratio of the inertial to viscous terms. Here, $D_{0}$ is the initial drop diameter, $U_{0}$ is the drop impact velocity, $\rho$ is the density of 
Hans-Jürgen Butt, Ilia V. Roisman, Martin Brinkmann, Periklis Papadopoulos, Doris Vollmer, Ciro Semprebon Current Opinion in Colloid \& Interf. Sci. (2014), 19, pp 343-354

the liquid, and $\eta$ is its viscosity. For water drops impacting on super-hydrophobic layers four different scenarios can be distinguished [103]:

- Deposition. For We roughly below 0.2 the drop is deposited on the surface. Impact dynamics of drops on surfaces can usually be divided into two phases. First, in the spreading phase the drop is flattened. In this process the kinetic energy is converted to interfacial energy of the deformed drop $[105,112,113]$. For low Weber numbers and thus low impact velocities the deformation of the drop is small as compared to its diameter.

- Rebound. With increasing Weber number the maximum lateral extension of the drop increases and the drop fully rebounces, as in example in Fig. 9. In most cases the appearance of the full rebound regime serves as an evidence of the surface hydrophobicity.

- Pinning/Sticking. At high We numbers the pressure underneath the drop exceeds the impalement pressure. The liquid penetrates into the superhydrophobic layer and goes to the Wenzel state. As a result the drop is pinned. The threshold velocity between rebound and pinning regimes depends on the specific repellency of the surface. This is the range where a test by drop impact is useful to characterize a layer.

- Splashing/fragmentation. At high Weber and Reynolds numbers, drops splash into many smaller droplets after impact (Fig. 10). It is known that the splashing threshold (which is the minimum impact velocity leading to splash) depends on the Reynolds and Weber numbers, surface roughness and on the air pressure [100, 101].

Among the main quantities which can be used to characterize the dynamic properties of super repellent layers are the maximum spreading diameter and the rebound/sticking threshold. 
Hans-Jürgen Butt, Ilia V. Roisman, Martin Brinkmann, Periklis Papadopoulos, Doris Vollmer, Ciro Semprebon Current Opinion in Colloid \& Interf. Sci. (2014), 19, pp 343-354

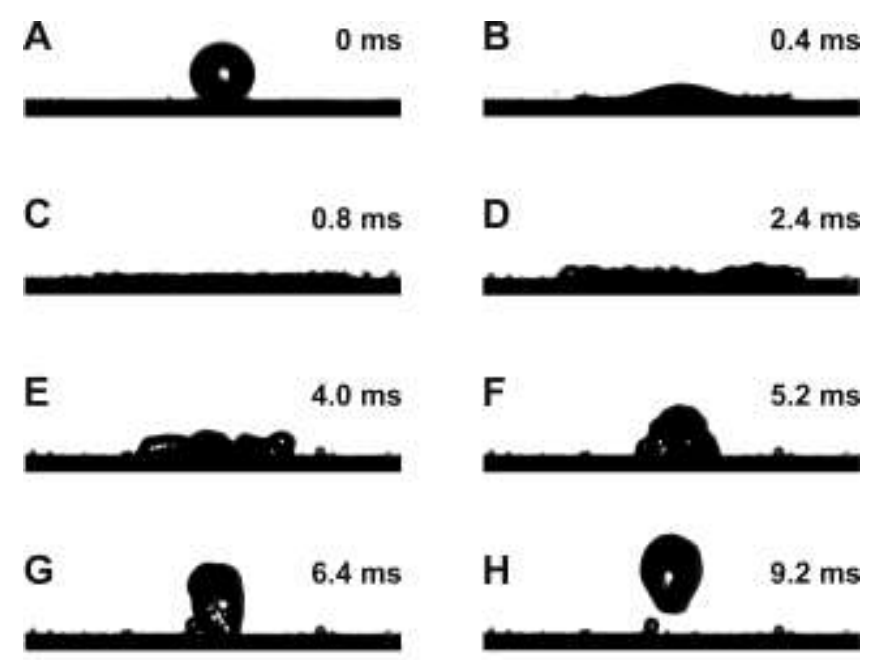

Figure 9. Impact and rebound of a $1.59 \mathrm{~mm}$ drop of water onto a cold $\left(-10^{\circ} \mathrm{C}\right)$ superhydrophobic layer with $3.3 \mathrm{~m} / \mathrm{s}$ impact velocity. The drop partially breaks up [121].

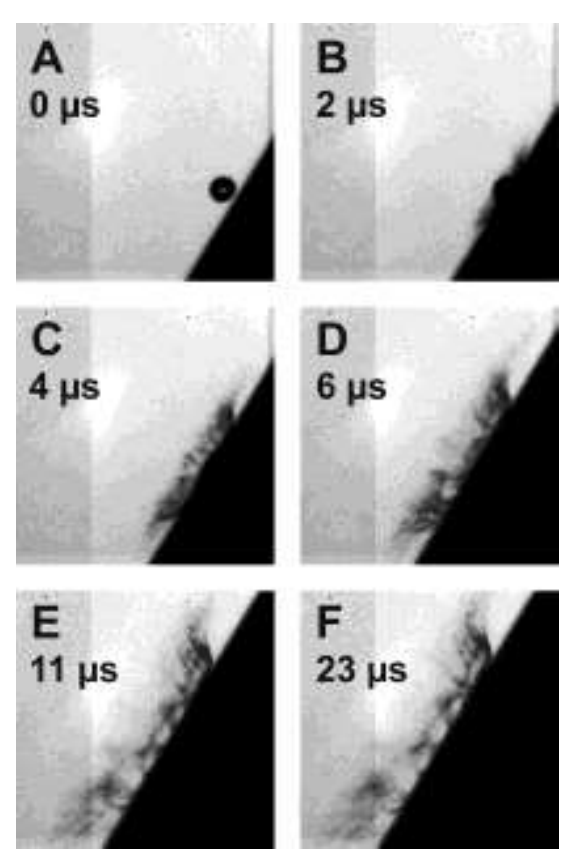

Figure 10. Splash of a drop of $194 \mu \mathrm{m}$ diameter on a superhydrophobic surface at an impact velocity $34 \mathrm{~m} / \mathrm{s}$ [121].

The Maximum drop spreading diameter $D_{\max }$ is relevant to many practical applications. Therefore, the ability of a certain surface to influence $D_{\max }$ could be an important characteristic of such surface. Clanet et al. [113] showed that the maximum spreading diameter on superhydrophobic substrates scales with the Weber number as $D_{\max } \propto W e^{1 / 4}$. 
Hans-Jürgen Butt, Ilia V. Roisman, Martin Brinkmann, Periklis Papadopoulos, Doris Vollmer, Ciro Semprebon Current Opinion in Colloid \& Interf. Sci. (2014), 19, pp 343-354

This scaling was validated by other experiments [114]. On the other hand, recent experiments [115] demonstrated (Fig. 11a), that at Weber numbers higher than $\approx 30$, the maximum spreading diameter does almost not depend on the substrate wettability. Even at Weber numbers smaller than 30 the difference between maximum spreading diameters on superhydrophobic and partially wettable substrates is not significant.

In fact, the maximum spreading diameter depends not only on the Weber number but also on the Reynolds number, since the role of viscosity on the spreading dynamics of the drop is important even for super liquid-repellant layers. One of the recently formulated expressions for the maximum spreading diameter for partially wettable substrates takes into account the evolution of the viscous boundary layer near the wall and the edge deceleration due to surface tension [116]:

$$
\frac{D_{\max }}{D_{0}}=0.87 R e^{1 / 5}-\beta \frac{R e^{2 / 5}}{W e^{1 / 2}}
$$

with $\beta=0.40$. Only the last term in Eq. (13) accounts for the forces associated with wettability. The corresponding coefficient can therefore be corrected if a drop impacting onto superhydrophobic layers is considered. By fitting experimental data, the corrected expression for superhydrophobic surfaces leads to $\beta=0.48$. A comparison of the predictions by Eq. (14) with the experimental data is shown in Fig. 11b. The agreement is rather good, which implies that high-speed impact onto superhydrophobic surfaces can be described by the same formalism as impact onto a smooth substrate. The difference is only in the magnitude of the force, associated with wettability, applied to the expanding edge of the drop. Therefore drop spreading at high Weber numbers cannot be used for substrate characterization since at these conditions the inertia is dominant while the effect of forces associated with surface tension and wettability is small. Surface characterization by measurements of the maximum spreading diameter has therefore to be performed only at small Weber numbers (We < 30) keeping the impact parameters constant for various substrates. 
Hans-Jürgen Butt, Ilia V. Roisman, Martin Brinkmann, Periklis Papadopoulos, Doris Vollmer, Ciro Semprebon Current Opinion in Colloid \& Interf. Sci. (2014), 19, pp 343-354
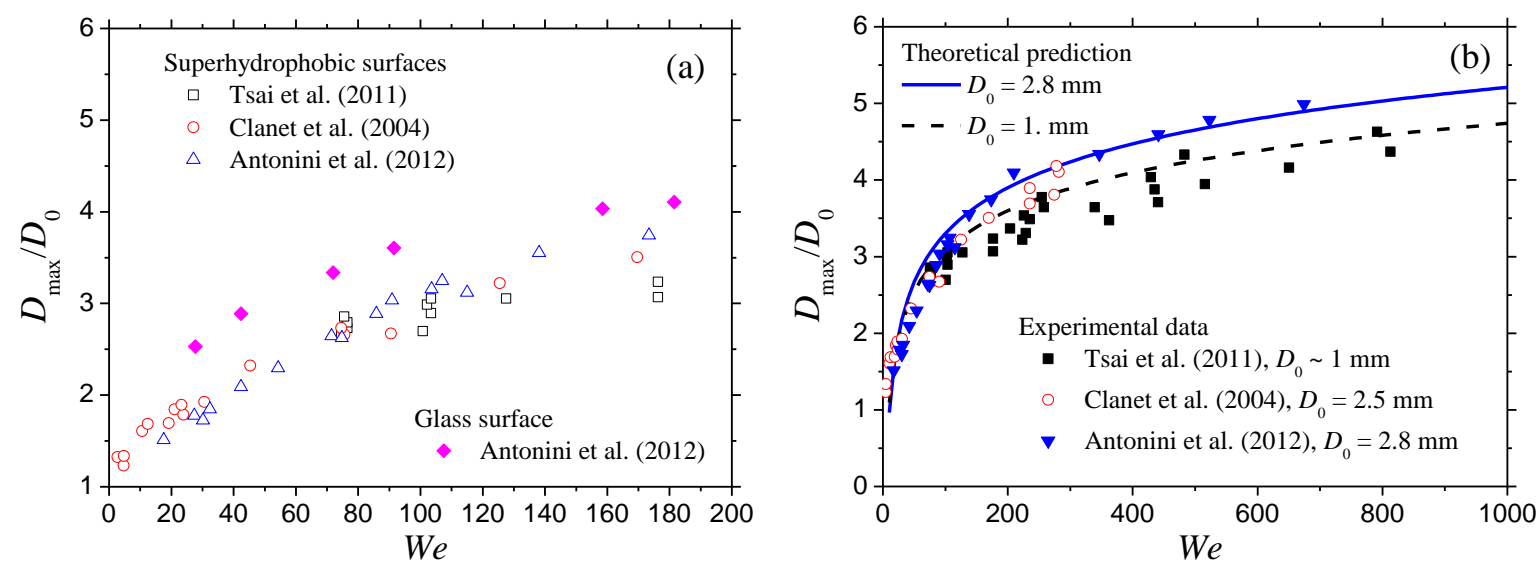

Figure 11. Maximal horizontal expansion of a water drop impacting onto a superhydrophobic substrate at relatively small Weber numbers (a) and high Weber numbers (b). Experimental [113-115] and values calculated with Eq. (13) and $\beta=0.48$ are compared for drops of initial diameter 1.0 and $2.8 \mathrm{~mm}$.

It should be noted that the rate of change of the drop diameter in the receding phase is mainly governed by surface tension and wettability [100]. This property can be used for characterization of the hysteresis of the contact angle of a certain substrate.

The Rebound/sticking threshold velocity is the minimum velocity for sticking, which is directly related to the impalement phenomenon. For drop impact the occurrence of the Cassie-to-Wenzel transition is often modeled by the average, static force balance, namely equating the maximum capillary pressure, which can be estimated for example from Eqs. (8)(10), to the dynamic pressure associated with impact $[102,117]$. This dynamic pressure is usually estimated from the stationary Bernoulli equation as $P_{d}=\rho U_{0}^{2} / 2$. For example, Jung \& Bhushan [105] estimate the critical impact velocity for the impalement on superhydrophobic layers consisting of an array of cylindrical pillars of the height $h$ and diameter $a$ as

$$
U_{c}=\frac{1}{d \sqrt{2}-a} \sqrt{\frac{32 \gamma h}{\rho}},
$$

where $d$ is the distance between the pillars. 
Hans-Jürgen Butt, Ilia V. Roisman, Martin Brinkmann, Periklis Papadopoulos, Doris Vollmer, Ciro Semprebon Current Opinion in Colloid \& Interf. Sci. (2014), 19, pp 343-354

In many cases such approaches successfully predict the critical velocity of the impalement despite the fact that some important assumptions, leading to the expressions of type (14), are not precise. First of all, the pressure produced by drop impact is not constant and is not precisely equal to $\rho U_{0}^{2} / 2$. At the very first instant (in fact of the relatively short duration of $D_{0} / c$, where $c$ is the speed of sound in the liquid) the pressure is produced by a shock of $P_{s}=\rho U_{0} c$ [118]. Afterwards the pressure quickly decreases. Numerical calculations show that the pressure at the impact region can be approximately expressed as

$$
P=1.7 \rho U_{0}^{2} \exp \left(-3.1 U_{0} t / D_{0}\right),
$$

(Fig. 12 in [119]). One important study which can help to understand the process of impalement of an impacting drop is the investigation of drop impact onto a thin porous membranes [120]. A drop impacting on such a porous membrane generates an array of small micro-jets behind the pore even at relatively small impact velocity as low as $2 \mathrm{~m} / \mathrm{s}$. They have estimated the penetration depth of the micro-jets in the pores of the membrane as

$$
h_{p} \approx \frac{\rho U_{0} d_{p}^{2}}{\eta}
$$

obtained from the balance of the inertial and viscous terms in the flow near the pore. Here, $d_{p}$ is the pore diameter.

The impalement velocity predicted using Eq. (14) and applied to the substrates consisting of the array of pillars is much smaller than that observed in experiments [105]. As we mentioned above, the critical velocity of impalement can be influenced by the entrapped air in the impact region preventing the liquid from contacting the substrate [114]. Sticking can occur when the time for drop spreading and receding is long enough for the diameter of the entrapped air lens to reach the receding drop diameter. The value of the impalement velocity (rebound/sticking threshold) is therefore a property of significant importance for characterizing the dynamic behavior of super liquid-repellent layers.

\section{Summary}


Hans-Jürgen Butt, Ilia V. Roisman, Martin Brinkmann, Periklis Papadopoulos, Doris Vollmer, Ciro Semprebon Current Opinion in Colloid \& Interf. Sci. (2014), 19, pp 343-354

The apparent receding contact angle $\Theta_{r}^{a p p}$ is the most important parameter characterizing the quality of a super liquid-repellent layers. Similar information is obtained when reporting the advancing $\Theta_{a}^{a p p}$ and the roll-off angle $\alpha(V)$ at defined drop volume. $\Theta_{r}^{a p p}, \Theta_{a}^{a p p}$, and $\alpha(V)$ represent the basic set of information, which allows comparing different layers. $\Theta_{r}^{a p p}$ and $\Theta_{a}^{a p p}$ are determined by the strength of contact line pinning for the receding and advancing edge of a drop, respectively. Microscopically the receding and advancing edge of drops progress in fundamentally different ways.

A useful phenomenological definition of super liquid-repellency with respect to a specific liquid is: $\Theta_{a}^{a p p} \geq 150^{\circ}$ and $\alpha \leq 10^{\circ}$ at a drop volume of $\pi \kappa^{3} / 6$ or typically $10 \mu \mathrm{L}$. For high contact angles it even suffices to report the apparent receding contact angle because it sets an upper limit for the roll-off angle. For example, a $5 \mu \mathrm{L}$ drop of water will roll-off for tilt angles $\leq 10^{\circ}$ for $\Theta_{r}^{a p p}=146^{\circ}$. For hexadecane the same condition leads to $\Theta_{r}{ }^{a p p}=136^{\circ}$.

We are still lacking theories which quantitatively link the microscopic structure of a layer to the apparent contact angles. Such a theory explicitly has to take into account that drops are usually not in a global thermodynamic equilibrium. Furthermore, for high contact angles the sessile drop method is often not precise. Therefore, complementary methods are important. Tensile (vertical) adhesion measurements are an additional, sensitive way of characterizing super liquid-repellency. They need to be carried out at defined boundary condition on the top plate, at defined liquid volume, and they need to be complemented by calculations taking into account the precise shape of the liquid surfaces. Vertical adhesion is linked to $\Theta_{r}^{a p p}$.

Measurements of the roll-off angle are linked to the apparent contact angle hysteresis. Carried out at defined drop volume they provide valuable information on the lateral adhesion. A universal theory, providing the proportionality factor $k$ between $\cos \Theta_{r}^{a p p}-\cos \Theta_{a}^{a p p}$ and $\sin \alpha$ is not yet available. It may not exist at all and $k$ may depend on the specific nano- and microstructure.

An important property characterizing the robustness of a super liquid-repellent layer is the impalement pressure. For low pressures, $\leq 3 \mathrm{kPa}$, the impalement pressure can be measured easily, e.g. by droop evaporation. Still no standardized method for testing more robust super 
Hans-Jürgen Butt, Ilia V. Roisman, Martin Brinkmann, Periklis Papadopoulos, Doris Vollmer, Ciro Semprebon Current Opinion in Colloid \& Interf. Sci. (2014), 19, pp 343-354

liquid-repellent layers is available. Drop impact is one semi-quantitative test, which is related to dynamic impalement.

\section{Acknowledgement}

HJB acknowledges financial support from the ERC grant 340391-SuPro.

\section{References}

[1] Dettre RH, Johnson RE. Contact angle hysteresis - porous surfaces. SCI Monograph 1967; 25:144-163.

[2] Barthlott W, Neinhuis C. Purity of the sacred lotus, or escape from contamination in biological surfaces. Planta 1997; 202:1-8.

[3] Onda T, Shibuichi S, Satoh N, Tsujii K. Super-water-repellent fractal surfaces. Langmuir 1996; 12:2125-2127.

[4] Chen W, Fadeev AY, Hsieh MC, Öner D, Youngblood J, McCarthy TJ. Ultrahydrophobic and ultralyophobic surfaces: Some comments and examples. Langmuir 1999; 15:3395-3399.

[5] Ma ML, Hill RM. Superhydrophobic surfaces. Curr. Op. Colloid Interface Sci. 2006; 11:193202.

[6] Herminghaus S. Roughness-induced non-wetting. Europhys. Lett. 2000; 52:165-170.

[7] Tuteja A, Choi W, Ma ML, Mabry JM, Mazzella SA, Rutledge GC et al. Designing superoleophobic surfaces. Science 2007; 318:1618-1622.

[8] Tuteja A, Choi W, Mabry JM, McKinley GH, Cohen RE. Robust omniphobic surfaces. Proc. Natl. Acad. Sci. USA 2008; 105:18200-18205.

[9] Dufour R, Harnois M, Thomy V, Boukherroub R, Senez V. Contact angle hysteresis origins: Investigation on super-omniphobic surfaces. Soft Matter 2011; 7:9380-9387.

[10] Im M, Im H, Lee JH, Yoon JB, Choi YK. A robust superhydrophobic and superoleophobic surface with inverse-trapezoidal microstructures on a large transparent flexible substrate. Soft Matter 2010; 6:1401-1404.

[11] Susarrey-Arce A, Marin AG, Nair H, Lefferts L, Gardeniers JGE, Lohse D et al. Absence of an evaporation-driven wetting transition on omniphobic surfaces. Soft Matter 2012; 8:97659770.

[12] Shibuichi S, Yamamoto T, Onda T, Tsujii K. Super water- and oil-repellent surfaces resulting from fractal structure. J. Colloid Interface Sci. 1998; 208:287-294.

[13] Steele A, Bayer I, Loth E. Inherently Superoleophobic Nanocomposite Coatings by Spray Atomization. Nano Letters 2009; 9:501-505.

[14] Xiong D, Liu GJ, Hong LZ, Duncan EJS. Superamphiphobic Diblock Copolymer Coatings. Chem. Mater. 2011; 23:4357-4366.

[15] Deng X, Mammen L, Butt H-J, Vollmer D. Candle soot as a template for a transparent robust superamphiphobic coating. Science 2012; 335:67-70.

[16] Yoshida E. Preparation of micro- and nanospheres with superamphiphobic surfaces by dispersion polymerization. Colloid Polym. Sci. 2012; 290:525-530.

[17] Nosonovsky M. Multiscale roughness and stability of superhydrophobic biomimetic interfaces. Langmuir 2007; 23:3157-3161. 
Hans-Jürgen Butt, Ilia V. Roisman, Martin Brinkmann, Periklis Papadopoulos, Doris Vollmer, Ciro Semprebon Current Opinion in Colloid \& Interf. Sci. (2014), 19, pp 343-354

[18] Joly L, Biben T. Wetting and friction on superoleophobic surfaces. Soft Matter 2009; 5:2549-2557.

[19] Watanabe K, Udagawa Y, Udagawa H. Drag reduction of Newtonian fluid in a circular pipe with a highly water-repellent wall. J. Fluid Mech. 1999; 381:225-238.

[20] McHale G, Newton MI, Shirtcliffe NJ. Immersed superhydrophobic surfaces: Gas exchange, slip and drag reduction properties. Soft Matter 2010; 6:714-719.

[21] Rothstein JP. Slip on superhydrophobic surfaces. Annu. Rev. Fluid Mech. 2010; 42:89109.

[22] Busse A, Sandham ND, McHale G, Newton MI. Change in drag, apparent slip and optimum air layer thickness for laminar flow over an idealised superhydrophobic surface. J. Fluid Mech. 2013; 727:488-508.

[23] Park KC, Chhatre SS, Srinivasan S, Cohen RE, McKinley GH. Optimal design of permeable fiber network structures for fog harvesting. Langmuir 2013; 29:13269-13277.

[24] Miljkovic N, Wang EN. Condensation heat transfer on superhydrophobic surfaces. MRS Bulletin 2013; 38:397-406.

[25] Paven M, Papadopoulos P, Schöttler S, Deng X, Mailänder V, Vollmer D et al. Super liquid-repellent gas membranes for carbon dioxide capture and heart-lung machines. Nature Commun. 2013; 4:2512.

[26] Xu QF, Mondal B, Lyons AM. Fabricating superhydrophobic polymer surfaces with excellent abrasion resistance by a simple lamination templating method. ACS Appl. Mater. Interfaces 2011; 3:3508-3514.

[27] Deng X, Mammen L, Zhao YF, Lellig P, Müllen K, Li C et al. Transparent, thermally stable and mechanically robust superhydrophobic surfaces made from porous silica capsules. Adv. Mater. 2011; 23:2962-2965.

[28] Zhou H, Wang HX, Niu HT, Gestos A, Wang XG, Lin T. Fluoroalkyl silane modified silicone rubber/nanoparticle composite: A super durable, robust superhydrophobic fabric coating. Adv. Mater. 2012; 24:2409-2412.

[29] Groten J, Rühe J. Surfaces with combined microscale and nanoscale structures: A route to mechanically stable superhydrophobic surfaces? Langmuir 2013; 29:3765-3772.

[30] Jin H, Tian X, Ikkala O, Ras RHA. Preservation of superhydrophobic and superoleophobic properties upon wear damage. ACS Appl. Mater. Interfaces 2013; 5:485-488.

[31] Gao LC, McCarthy TJ. Teflon is hydrophilic. Comments on definitions of hydrophobic, shear versus tensile hydrophobicity, and wettability characterization. Langmuir 2008; 24:9183-9188.

[32] Kota AK, Kwon G, Choi W, Mabry JM, Tuteja A. Hygro-responsive membranes for effective oil-water separation. Nature commun. 2012; 1038:1-7.

[33] Barthlott W. Die Selbstreinigungsfähigkeit pflanzlicher Oberflächen durch Epicuticularwachse. In: Verantwortung für die Zukunft. Klima- und Umweltforschung an der Universität Bonn. Bonn: 1992. pp. 117-120.

[34] Wayner PC. The interfacial profile in the contact line region and the Young-Dupré equation. J. Colloid Interface Sci. 1982; 88:294-295.

[35] Churaev NV, Starov VM, Derjaguin BV. The shape of the transition zone between a thin film and bulk liquid and the line tension. J. Colloid Interface Sci. 1982; 89:16-24.

[36] de Gennes PG. Wetting: Statics and dynamics. Rev. Modern Phys. 1985; 57:827-863. 
Hans-Jürgen Butt, Ilia V. Roisman, Martin Brinkmann, Periklis Papadopoulos, Doris Vollmer, Ciro Semprebon Current Opinion in Colloid \& Interf. Sci. (2014), 19, pp 343-354

[37] Kleingartner JA, Srinivasan S, Mabry JM, Cohen RE, McKinley GH. Utilizing dynamic tensiometry to quantify contact angle hysteresis and wetting state transitions on nonwetting surfaces. Langmuir 2013; 29:13396-13406.

[38] Drelich J. Guidelines to measurements of reproducible contact angles using a sessiledrop technique. Surface Innovations 2013; 4:248-254.

[39] Extrand CW, Moon SI. Contact angles of liquid drops on super hydrophobic surfaces: Understanding the role of flattening of drops by gravity. Langmuir 2010; 26:17090-17099.

[40] Korhonen JT, Huhtamaki T, Ikkala O, Ras RHA. Reliable measurement of the receding contact angle. Langmuir 2013; 29:3858-3863.

[41] Papadopoulos P, Deng X, Mammen L, Drotlef DM, Battagliarin G, Li C et al. Wetting on the Microscale: Shape of a Liquid Drop on a Microstructured Surface at Different Length Scales. Langmuir 2012; 28:8392-8398.

[42] Papadopoulos P, Mammen L, Deng X, Vollmer D, Butt HJ. How superhydrophobicity breaks down. Proc. Natl. Acad. Sci. USA 2013; 110:3254-3258.

[43] Wiegand G, Neumaier KR, Sackmann E. Microinterferometry: three-dimensional reconstruction of surface microtopography for thin-film and wetting studies by reflection interference contrast microscopy (RICM). Applied Optics 1998; 37:6892-6905.

[44] Fischer DG, Ovryn B. Interfacial shape and contact-angle measurement of transparent samples with confocal interference microscopy. Optics Lett. 2000; 25:478-480.

[45] Callies M, Quéré D. On water repellency. Soft Matter 2005; 1:55-61.

[46] Starov VM, Velarde MG, Radke CJ. Wetting and Spreading Dynamics. London: CRC Press; 2007. 515 p.

[47] Butt HJ, Semprebon C, Papadopoulos P, Vollmer D, Brinkmann M, Ciccotti M. Design principles for superamphiphobic surfaces. Soft Matter 2013; 9:418-428.

[48] Cassie ABD, Baxter S. Wettability of porous surfaces. Trans. Faraday Soc. 1944; 40:546551.

[49] Bico J, Marzolin C, Quéré D. Pearl drops. Europhys. Lett. 1999; 47:220-226.

[50] Dorrer C, Rühe J. Some thoughts on superhydrophobic wetting. Soft Matter 2009; 5:5161.

[51] Öner D, McCarthy TJ. Ultrahydrophobic surfaces. Effects of topography length scales on wettability. Langmuir 2000; 16:7777-7782.

[52] Yoshimitsu Z, Nakajima A, Watanabe T, Hashimoto K. Effects of surface structure on the hydrophobicity and sliding behavior of water droplets. Langmuir 2002; 18:5818-5822.

[53] Extrand CW. Model for contact angles and hysteresis on rough and ultrahydrophobic surfaces. Langmuir 2002; 18:7991-7999.

[54] Gao L, McCarthy TJ. How Wenzel and Cassie were wrong. Langmuir 2007; 23:3762-3765. [55] Choi W, Tuteja A, Mabry JM, Cohen RE, McKinley GH. A modified Cassie-Baxter relationship to explain contact angle hysteresis and anisotropy on non-wetting textured surfaces. J. Colloid Interface Sci. 2009; 339:208-216.

[56] Paxson AT, Varanasi KK. Self-similarity of contact line depinning from textured surfaces. Nature commun. 2013; 4:1492-1496.

[57] Dorrer C, Rühe J. Contact line shape on ultrahydrophobic post surfaces. Langmuir 2007; 23:3179-3183.

[58] Mognetti BM, Yeomans JM. Modeling receding contact lines on superhydrophobic surfaces. Langmuir 2010; 26:18162-18168. 
Hans-Jürgen Butt, Ilia V. Roisman, Martin Brinkmann, Periklis Papadopoulos, Doris Vollmer, Ciro Semprebon Current Opinion in Colloid \& Interf. Sci. (2014), 19, pp 343-354

[59] Semprebon C, Herminghaus S, Brinkmann M. Advancing modes on regularly patterned substrates. Soft Matter 2012; 8:6301-6309.

[60] Reyssat $M$, Quéré $D$. Contact angle hysteresis generated by strong dilute defects. J. Phys. Chem. B 2009; 113:3906-3909.

[61] Samuel B, Zhao H, Law KY. Study of wetting and adhesion interactions between water and various polymer and superhydrophobic surfaces. J. Phys. Chem. C 2011; 115:1485214861.

[62] Pilat DW, Papadopoulos P, Schäffel D, Vollmer D, Berger R, Butt H-J. Dynamic measurement of the force required to move a liquid drop on a solid surface. Langmuir 2012; 28:16812-16820.

[63] Zhang JH, Wang JM, Zhao Y, Xu L, Gao XF, Zheng YM et al. How does the leaf margin make the lotus surface dry as the lotus leaf floats on water. Soft Matter 2008; 4:2232-2237.

[64] Ensikat HJ, Mayser M, Barthlott W. Superhydrophobic and adhesive properties of surfaces: Testing the quality by an elaborated scanning electron microscopy method. Langmuir 2012; 28:14338-14346.

[65] Cheng Z, Lai H, Du M, Zhu S, Zhang N, Sun K. Super-hydrophobic surface with switchable adhesion responsive to both temperature and pH. Soft Matter 2012; 8:9635-9641.

[66] Ye M, Deng X, Ally J, Papadopoulos P, Schellenberger F, Vollmer D et al. Superamphiphobic particles - how small can we go? Phys. Rev. Lett. 2014; 112:016101.

[67] Furmidge CGL. Studies at phase interfaces. I. The sliding of liquid drops on solid surfaces and a theory for spray retention. J. Colloid Sci. 1962; 17:309-324.

[68] Schrader ME. Young-Dupre revisited. Langmuir 1995; 11:3585-3589.

[69] Baret JC, Brinkmann M. Wettability control of droplet deposition and detachment. Phys. Rev. Lett. 2006; 96:146106.

[70] Butt HJ, Kappl M. Normal capillary forces. Adv. Colloid Interface Sci. 2009; 146:48-60.

[71] Nakajima A. Control of static and dynamic hydrophobicity of solid surface and its application. J. Ceramic Soc. Japan 2011; 119:711-719.

[72] Macdougall G, Ockrent C. Surface energy relations in liquid/solid systems. I. The adhesion of liquids to solids and a new method of determining the surface tension of liquids. Proc. Roy. Soc. London A 1942; 180:151-173.

[73] Kawasaki K. Study of wettability of polymers by sliding of a water drop. J. Colloid Sci. 1960; 15:402-407.

[74] Frenkel YI. On the behavior of liquid drops on a solid surface. 1. The sliding of drops on an inclined surface. J. Exptl. Theoret. Phys. (USSR) 1948; 18:659-669.

[75] Dussan EB. On the ability of drops or bubbles to stick to non-horizontal surfaces of solids. 2. Small drops or bubbles having contact angles of arbitrary size. J. Fluid Mech. 1985; 151:1-20.

[76] Quéré D, Azzopardi MJ, Delattre L. Drops at rest on a tilted plane. Langmuir 1998; 14:2213-2216.

[77] Sakai M, Song JH, Yoshida N, Suzuki S, Kameshima Y, Nakajima A. Direct observation of internal fluidity in a water droplet during sliding on hydrophobic surfaces. Langmuir 2006; 22:4906-4909.

[78] ElSherbini A, Jacobi A. Retention forces and contact angles for critical liquid drops on non-horizontal surfaces. J. Colloid Interface Sci. 2006; 299:841-849. 
Hans-Jürgen Butt, Ilia V. Roisman, Martin Brinkmann, Periklis Papadopoulos, Doris Vollmer, Ciro Semprebon Current Opinion in Colloid \& Interf. Sci. (2014), 19, pp 343-354

[79] Antonini C, Carmona FJ, Pierce E, Marengo M, Amirfazli A. General methodology for evaluating the adhesion force of drops and bubbles on solid surfaces. Langmuir 2009; 25:6143-6154.

[80] Extrand CW. Designing for optimum liquid repellency. Langmuir 2006; 22:1711-1714.

[81] Brown RA, Orr FM, Scriven LE. Static drop on an inclined plane: Analysis by the finite element method. J. Colloid Interface Sci. 1980; 73:76-87.

[82] Extrand CW, Kumagai Y. Liquid drops on an inclined plane: The relation between contact angles, drops hape, and retention forces. J. Colloid Interface Sci. 1995; 170:515-521.

[83] Wolfram E, Faust R. Liquid drops on a tilted plate, contact angle hysteresis and the Young contact angle. In: Wetting, Spreading and Adhesion. Padday JF (Editor) New York: Academic Press; 1978. pp. 213-222.

[84] Krasovitski B, Marmur A. Drops down the hill: Theoretical study of limiting contact angles and the hysteresis range on a tilted plate. Langmuir 2005; 21:3881-3885.

[85] Pierce E, Carmona FJ, Amirfazli A. Understanding of sliding and contact angle results in tilted plate experiments. Colloids \& Surfaces A 2008; 323:73-82.

[86] Semprebon C, Brinkmann M. On the onset of motion of sliding drops Soft Matter 2014; 10:3325-3334.

[87] Lafuma A, Quéré D. Superhydrophobic states. Nature Materials 2003; 2:457-460.

[88] Reyssat M, Yeomans JM, Quéré D. Impalement of fakir drops. Europhys. Lett. 2008; 81:26006.

[89] McHale G, Aqil S, Shirtcliffe NJ, Newton MI, Erbil HY. Analysis of droplet evaporation on a superhydrophobic surface. Langmuir 2005; 21:11053-11060.

[90] Kwon DH, Lee SJ. Impact and wetting behaviors of impinging microdroplets on superhydrophobic textured surfaces. Appl. Phys. Lett. 2012; 100:171601.

[91] Hensel R, Helbig R, Aland S, Braun HG, Voigt A, Neinhuis C et al. Wetting resistance at its topographical limit: The benefit of mushroom and serif $\mathrm{T}$ structures. Langmuir 2013; 29:1100-1112.

[92] Checco A, Ocko BM, Tasinkevych M, Dietrich S. Stability of thin wetting films on chemically nanostructured surfaces. Phys. Rev. Lett. 2012; 109:166101.

[93] Lobaton EJ, Salamon TR. Computation of constant mean curvature surfaces: Application to the gas-liquid interface of a pressurized fluid on a superhydrophobic surface. J. Colloid Interface Sci. 2007; 314:184-198.

[94] Kusumaatmaja H, Blow ML, Dupuis A, Yeomans JM. The collapse transition on superhydrophibic surfaces. Eur. Phys. Lett. 2008; 81:36003.

[95] Bormashenko E, Musin A, Whyman G, Zinigrad M. Wetting Transitions and Depinning of the Triple Line. Langmuir 2012; 28:3460-3464.

[96] Poetes R, Holtzmann K, Franze K, Steiner U. Metastable underwater superhydrophobicity. Phys. Rev. Lett. 2010; 105:166104.

[97] Moulinet S, Bartolo D. Life and death of a fakir droplet: Impalement transitions on superhydrophobic surfaces. Eur. Phys. J. E 2007; 24:251-260.

[98] Biance AL, Clanet C, Quéré D. First steps in the spreading of a liquid droplet. Phys. Rev. E 2004; 69:016301.

[99] Gnanappa AK, Papageorgiou DP, Gogolides E, Tserepi A, Papathanasiou AG, Boudouvis AG. Hierarchical, plasma nanotextured, robust superamphiphobic polymeric surfaces structurally stabilized through a wetting-drying cycle. Plasma Process. Polym. 2012; 9:304315. 
Hans-Jürgen Butt, Ilia V. Roisman, Martin Brinkmann, Periklis Papadopoulos, Doris Vollmer, Ciro Semprebon Current Opinion in Colloid \& Interf. Sci. (2014), 19, pp 343-354

[100] Yarin AL. Drop impact dynamics: Splashing, spreading, receding, bouncing... Annu. Rev. Fluid Mech. 2006; 38:159-192.

[101] Marengo M, Antonini C, Roisman IV, Tropea C. Drop collisions with simple and complex surfaces. Curr. Op. Colloid Interface Sci. 2011; 16:292-302.

[102] Bartolo D, Bouamrirene F, Verneuil E, Buguin A, Silberzan P, Moulinet S. Bouncing or sticky droplets: Impalement transitions on superhydrophobic micropatterned surfaces. Europhys. Lett. 2006; 74:299-305.

[103] Rioboo R, Voué $M$, Vaillant A, De Coninck J. Drop impact on porous superhydrophobic polymer surfaces. Langmuir 2008; 24:14074-14077.

[104] Kwak G, Lee M, Senthil K, Yong K. Impact dynamics of water droplets on chemically modified $\mathrm{WO}_{\mathrm{x}}$ nanowire arrays. Appl. Phys. Lett. 2009; 95:153101.

[105] Jung YC, Bhushan B. Dynamic effects of bouncing water droplets on superhydrophobic surfaces. Langmuir 2008; 24:6262-6269.

[106] Nguyen TPN, Brunet P, Coffinier Y, Boukherroub R. Quantitative Testing of Robustness on Superomniphobic Surfaces by Drop Impact. Langmuir 2010; 26:18369-18373.

[107] Tsai P, van der Veen RCA, van de Raa M, Lohse D. How Micropatterns and Air Pressure Affect Splashing on Surfaces. Langmuir 2010; 26:16090-16095.

[108] Duvivier D, Rioboo R, Voue M, De Coninck J. Drop impact on superhydrophobic surfaces - varying gravitational effects. Atomization and Sprays 2012; 22:409-429.

[109] Patankar NA. Consolidation of hydrophobic transition criteria by using an approximate energy minimization approach. Langmuir 2010; 26:8941-8945.

[110] Pan S, Kota AK, Mabry JM, Tuteja A. Superomniphobic surfaces for effective chemical shielding. J. Am. Chem. Soc. 2013; 135:578-581.

[111] Deng X, Schellenberger F, Papadopoulos P, Vollmer D, Butt HJ. Liquid drops impacting superamphiphobic coatings. Langmuir 2013; 29:7847-7856.

[112] Richard D, Clanet C, Quéré D. Surface phenomena - Contact time of a bouncing drop. Nature 2002; 417:811-811.

[113] Clanet C, Béguin C, Richard D, Quéré D. Maximal deformation of an impacting drop. J. Fluid Mech. 2004; 517:199-208.

[114] Tsai P, Hendrix MHW, Dijkstra RRM, Shui L, Lohse D. Microscopic structure influencing macroscopic splash at high Weber number. Soft Matter 2011; 7:11325-11333.

[115] Antonini C, Amirfazli A, Marengo M. Drop impact and wettability: From hydrophilic to superhydrophobic surfaces. Phys. Fluids 2012; 24:102104.

[116] Roisman IV. Inertia dominated drop collisions. II. An analytical solution of the NavierStokes equations for a spreading viscous film. Phys. Fluids 2009; 21:052104.

[117] Reyssat M, Pépin A, Marty F, Chen Y, Quéré D. Bouncing transitions on microtextured materials. Europhys. Lett. 2006; 74:306-312.

[118] Lembach AN, Tan HB, Roisman IV, Gambaryan-Roisman T, Zhang YY, Tropea C et al. Drop Impact, Spreading, Splashing, and Penetration into Electrospun Nanofiber Mats. Langmuir 2010; 26:9516-9523.

[119] Roisman IV, Berberovic E, Tropea C. Inertia dominated drop collisions. I. On the universal flow in the lamella. Phys. Fluids 2009; 21:052103.

[120] Sahu RP, Sinha-Ray S, Yarin AL, Pourdeyhimi B. Drop impacts on electrospun nanofiber membranes. Soft Matter 2012; 8:3957-3970.

[121] Li H. Drop impact on dry surfaces with phase change. In: Mechanical Engineering. Darmstadt: Technische Universität Darmstadt; 2013. 
Hans-Jürgen Butt, Ilia V. Roisman, Martin Brinkmann, Periklis Papadopoulos, Doris Vollmer, Ciro Semprebon Current Opinion in Colloid \& Interf. Sci. (2014), 19, pp 343-354 